\section{Comparison of Biological and Chemical Control of Greenhouse Whitefly on Poinsettia Stock Plants}

\author{
R.W. McMahon ${ }^{1}$, \\ R.K. Lindquist ${ }^{2}$, A.C. Witt ${ }^{3}$, \\ S.H. Kinnamon ${ }^{4}$, and \\ M.L. Casey $^{2}$
}

Additional index words. Euphorbia pulcherrima Wild., Encarsia formosa Gahan, Trialeurodes vaporariorum West-wood, floriculture

Summary. A demonstration study was conducted to compare the effectiveness of biological and chemical control treatments on the greenhouse whitefly (GHWF) (Trialeurodes vaporariorum, Westwood) using poinsettia (Euphorbia pulcherrima Wild.) stock plants. Two identical greenhouse compartments, each containing 84 stock plants, were used. In the biological control compartment, three biweekly releases of Encarsia formosa (EF) were made, while in the chemical control compartment eight weekly applications of resmethrin or acephate aerosol treatments were made. Results showed that overall greenhouse whitefly populations in the chemical control compartment were slightly lower than in the biological control compartment. Cuttings taken from stock plants in the biological control compartment at the end of the experiment were commercially acceptable with regard to the presence of GHWF adults. Chemical names used: O,S-dimethyl acetylphosphoramidothioate (acephate), [5-(phenylmethyl)-3-furanyl]

Ohio Agricultural Research and Development Center, 1680 Madison Avenue, Wooster, OH 44691.

${ }^{1}$ Agricultural Technical Institute, 1328 Dover Road, Wooster, OH 44691.

${ }^{2}$ Dept. of Entomology, Ohio Agricultural Research and Development Center, 1680 Madison Avenue, Wooster, $\mathrm{OH} 44691$.

${ }^{3} 3673$ Fenley Road, Cleveland Heights, OH 44121.

${ }^{4} 212$ Pershing Avenue, Galion, $\mathrm{OH} 44833$ methyl 2,2-dimethyl-3-(2-methyl-1propenyl)cyclopropane-carboxylate (resmethrin).

B iological control of the greenhouse whitefly (GHWF) by the parasitic wasp Encarsia formosa (EF) has been implemented in the greenhouse vegetable industry throughout the world for many years (Delorme et al., 1985; Van Roermund and Van Lenteren, 1990). The GHWF is also a pest of many greenhouse floriculture crops (McHugh, 1991 a). However, the use of EF for controlling GHWF in the floriculture industry is rare at present (Parrella, 1990).

There are several reasons that biological control has not been practiced extensively in the floriculture industry. These reasons include an extremely low tolerance for the presence of pests and their damage on floriculture crops and the effective use of pesticides to achieve this goal (McMahon et al., 1989; Parrella, 1990). However, pesticide resistance of many pests, including GHWF (McHugh, 1991 b), is increasing rapidly, making chemical control of greenhouse pests more difficult. In addition, many pesticides have been and are being removed from use by pesticide regulations. The cost of developing new pesticides is very high and this greatly slows the release of new pesticides for use in the industry (Cooper and Oetting, 1987). In addition, the public is becoming increasingly concerned about the use of pesticides on floriculture crops (Heinz and Parrella, 1990).

For these reasons, biological control of pests in the floriculture industry is becoming more attractive as reliance on conventional pesticide use decreases. One of the floriculture crops most suitable for biological control of pests is the poinsettia (McMahon et al., 1989; Parrella, 1990). This crop often is grown as a monoculture and, as such, avoids the complications of pest control experienced when two or more crops with different associated pests are grown in the same greenhouse. Another reason is that poinsettias have only two serious pests: the GHWF and the sweetpotato whitefly (Ecke et al. 1990). Therefore, implementing biological control measures on a poinsettia crop should be relatively simple, in contrast to other greenhouse crops.
There are several reports in the literature on the effective use of EF for biological control of GHWF on poinsettia Christmas crops (Albert, 1990; Benuzzi et al., 1990; McMahon et al., 1989; Michael, 1991). Weekly releases of EF beginning at the start of the crop (when GHWF populations were low) resulted in GHWF control comparable to chemical control treatments. EF actively searched for and parasitized GHWF larval stages or nymphs by laying eggs within the GHWF nymphs. The EF larvae consumed the parasitized nymphs during development and emerged as adult EF to continue this cycle.

Another phase of poinsettia production for many growers is the late spring and summer production of stock plants, from which cuttings are taken for the Christmas crop. Like the Christmas crop, poinsettia stock plants are also prone to GHWF infestation and they require pesticide treatments in most cases. The objective of this study, therefore, was to evaluate $\mathrm{EF}$ in the biological control of GHWF on poinsettia stock plants.

The experiment was conducted in two identical $7 \times 7-\mathrm{m}$ compartments. Rootedcuttings of four Annette Hegg poinsettia cultivars- Diva Starlight, Hot Pink, Lady, and Brilliant Diamond -were used. The cuttings were planted in $20.5-\mathrm{cm}$ azalea pots on 23 May 1989. Standard poinsettia stock plant culture was followed as described by Ecke et al. (1990).

The plants were arranged in three blocks per compartment using a randomized complete- block design. Each block contained seven plants per cultivar, for a total of 84 plants per compartment. Stock plants were placed on inverted $25.5-\mathrm{cm}$ azalea pots and grown on $61-\mathrm{cm}$ centers. Three days after planting, 168 GHWF adults (an average of two GHWF per plant) were released from the center of both compartments.

Two weeks after releasing the GHWF, three biweekly releases of EF (Kopper \& Company, North Ridgeville, Ohio) began on 9 June 1989 in the biological control compartment. Small cards $(4 \times 6 \mathrm{~cm})$, each with -70 parasitized GHWF nymphs glued to it, were placed within the plant canopy at several locations throughout the compartment. Based on 70 parasitized nymphs per card, an average of 9.5 adult EF per plant were released each 
time, for a total of 28.5 adult EF per plant (Table 1.) These figures are based on the assumption that $100 \%$ of the parasitized nymphs produced an adult EF.

The chemical control treatments in the other compartment consisted of eight weekly aerosol sprays applying either resmethrin or acephate (Table $1)$. These pesticides were applied at the labeled rate in the late afternoon by directing the sprays slightly above the plant canopy while walking through the compartment. During application, ventilation fans were off and the vents were closed and remained so overnight.

Twovertical $5 \times 5$-cm yellow sticky traps per block were placed at plant canopy level, from which the number of trapped GHWF were recorded weekly. Population counts were taken biweekly from two plants per cultivar per block. The number of GHWF adults and nymphs, EF, and parasitized GHWF nymphs were recorded. The data within each compartment were analyzed by analysis of variance with means separation by LSD $(P=0.05)$.

Poinsettia cultivar did not have an effect on GHWF nymph parasitism by EF nor on immature and mature GHWF populations in both compartments. This is in agreement with other findings (McMahon et al., unpublished data).

EF became established in the biological control compartment between their second and third releases (4 weeks after their first release), as evidenced by $32 \%$ parasitism of the GHWF nymphs on 6 July (Fig. 1A). GHWF nymph parasitism increased to $66 \%$ on 20 July and remained at $66 \%$ on 3 Aug.

Table 1. Release rates of natural and chemical control schedule for the poinsettia stock plants.

Date $\quad \frac{\text { Biological control compartment }}{\text { Encarsia formosa (avg. no./plant) }} \quad \frac{\text { Chemical control compartment }}{\text { Pesticide }^{2}}$

8 June

9 June

19 June

22 June

23 June

30 June

8 July

9 July

15 July

21 July

29 July

Total Escarsia
A small population of EF also became established in the chemical control compartment 2 weeks after the first EF release in the other compartment. In the chemical control compartment, $18 \%$ to $22 \%$ of the GHWF nymphs were parasitized from 22 June to 3 Aug. (Fig. 1B).

Biweekly population counts showed that the number of GHWF adults per plant were higher in the biological control house than in the chemical control house on all sampling dates except 20 July. There was an increase in the average number of GHWF adults per plant, from 3 on 22 June to 8.1 on 6 July (Fig. 1A). The average number of GHWF adults per plant then declined to 1.25 on 20 July and increased to 3 on 3 Aug. In the chemical control compartment, 1.2 GHWF adults per plant were present on 22 June and decreased to a low of 0.20 on 6 July and did not exceed either of these limits for the rest of the experiment (Fig. 1B).

The total number of GHWF nymphs per plant in the biological control compartment increased from 3 on 22 June to 60 on 6 July (Fig. 1A). The nymph population peaked 2 weeks later at 75 and decreased to 61 on 3 Aug. In the chemical control compartment, the average number of GHWF nymphs per plant was lower than the biological control compartment for all sampling dates. On 22 June, 4 GHWF nymphs per plant were present, followed by an increase to 9.5 on 20 July and a decrease to 8.5 at the end of the experiment on $3 \mathrm{Aug}$. (Fig. 1B).

Yellow sticky trap data showed

${ }^{\bar{z}}$ Applied as aerosol formulations.

${ }^{y}$ Resmethrin (PT 1200 ${ }^{\circledR}$ ); Acephate (PT $1300^{\circledR}$ ), Whitmire Laboratories, Inc., St. Louis, Mo. that more GHWF adults were caught per trap in the biological control compartment for the entire S-week period (Fig. 2). On 6 July, the first sampling date, an average of 30 GHWF adults were counted on each trap in the biological control compartment and, on 13 July, an average of 38 GHWF adults per trap were counted (Fig. 2). The number of GHWF adults per trap then declined to 19 at the end of the experiment. In the chemical control compartment, the number of GHWF adults caught per trap remained at 5 or lower during the S-week period (Fig. 2).

The results of this experiment show that EF can become established on poinsettia stock plants and can control GHWF populations during a relatively short time period when released at a rate of 28.5 per plant. The increase in GHWF adult population in the biological control compartment from 22 June to 6 July was followed by a substantial decrease in population during the next 2 weeks. This occurred 4 to 6 weeks after the first EF release.

This population decrease corresponded with an increase of GHWF nymph parasitism from $32 \%$ to $66 \%$ (Fig. 1). Similar results were reported by Michael (1991) on a poinsettia Christmas crop, with 24 EF per plant (released over an S-week period), except that parasitism approached $80 \%$. However, Benuzzi et al. (1990) achieved effective biological control of GHWF on poinsettias when EF parasitized $61 \%$ to $64 \%$ of the GHWF nymphs, similar to results of this experiment. There was an increase in the GHWF adult population at the end of the experiment to 3 per plant, which was the same number per plant on 22 June, when population counts were first taken. This GHWF population is very similar those observed by Benuzzi et al. (1990). They reported that EF maintained a level of 2 to 4 GHWF per poinsettia during the last 5 weeks. The net result of the EF in this experiment was an increase of $1 \mathrm{GHWF}$ to 3 per plant on 3 Aug. after the initial release of 2 GHWF per plant on 26 May.

Gillespie and Quiring (1987) reported that a density of 10 yellow sticky traps $(60 \times 4 \mathrm{~cm})$ per 180 tomato plants resulted in major reductions of GHWF populations. In our study, yellow sticky traps were placed at a density of one trap per 14 plants. Although these traps were only one- 
A. Biocontrol Compartment



B. Chemical Control Compartment

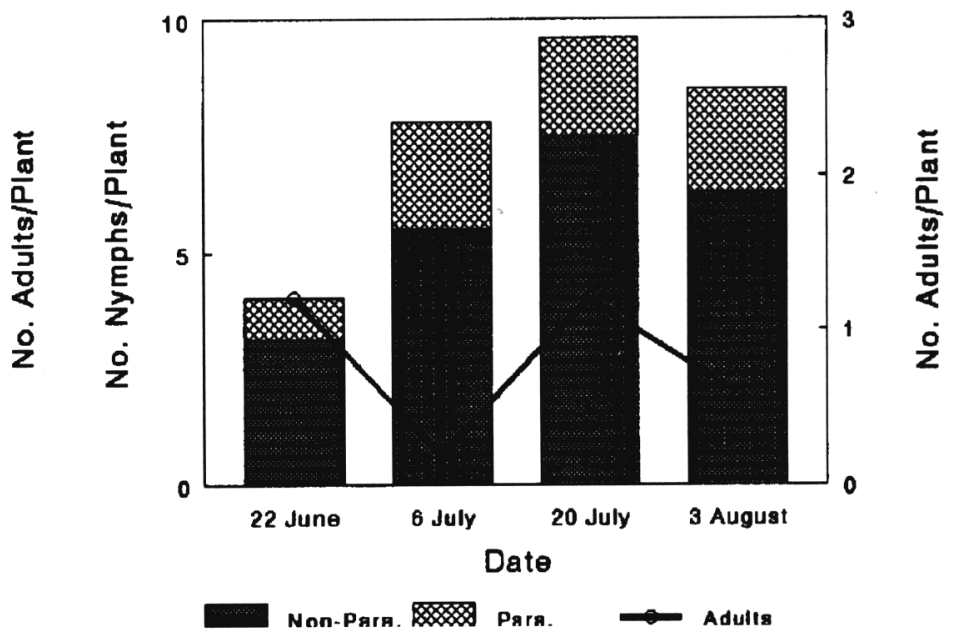

Fig. 1. A \& B. Number of parasitized and nonparasitizd greenhouse whitefly nymphs per plant and the number of geenhouse whitefly adults per plant in the biological and chemical control compartments.

tenth the size of those used by Gillespie and Quiring (1987), the trap density was more than 10 times as great. Thus, the yellow sticky traps also may have contributed to the decline in GHWF adults caught in the biological control compartment from 6 July to 3 Aug.

The eight aerosol applications of acephate and resmethrin effectively controlled immature and adult GHWF populations throughout the experiment. Considerably lower populations of both GHWF nymph and adult stages were recorded in the chemical control house than in the biological control house. The number of GHWF adults caught per yellow sticky trap also was lower in the chemical control house. This lower number may be due in part to the fact that EF also became established in the chemical control compartment despite eight weekly pesticide applications. EF migrated from the biological control compartment across a 2-m-wide corridor into the chemical control house. Since $22 \%$ of the GHWF nymphs were parasitized, this would lead to a corresponding decrease in emerging GHWF adults. These results suggest that resmethrin and acephate aerosol applications could be used, if necessary, in a biological control program using EF without totally disrupting EF.

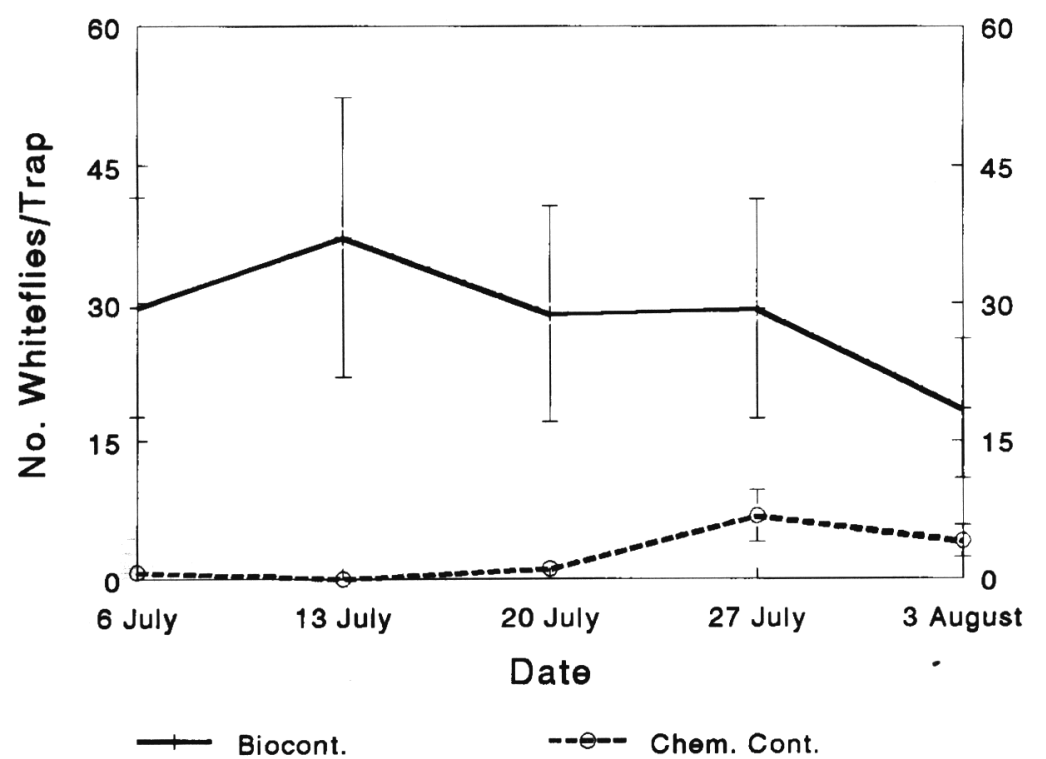

Fig. 2. Number of greenhouse whitefly adults per yellow sticky trap in the biological and chemical control compartments. Vertical bars indicate the SE of six yellow sticky traps.
A cost analysis was performed for a 1 -acre greenhouse area based on the rate of pesticide and EF applications made in this study and on the current prices of EF, acephate, and resmethrin. The cost of chemical control for this area would be about $\$ 800$ compared to $\$ 2180$ for biological control using EF. Labor costs are not included in these figures, as we estimated these costs to be similar in both situations. The release rate of $28.5 \mathrm{EF}$ per plant is much higher than in a later study in which effective biological control of SPWF was obtained at a total release rate of $6 \mathrm{EF}$ per plant on a Christmas poinsettia crop (McMahon et al., unpublished data). If such a release rate were implemented for the poinsettia stock plants, then the cost of $\mathrm{EF}$ for controlling GHWF would be $\$ 460$, which would compare favorably with chemical control costs. However, because poinsettia stock plants are larger than the Christmas crop, a release rate greater than 6 would probably have to be applied. A realistic rate of $12 \mathrm{EF}$ per stock plant would cost a grower $\$ 920$ for an acre, which would still compare favorably with chemical control costs.

At the end of the study, terminal stem cuttings were taken from the stock plants produced in the biological and chemical control compartments. As the cuttings were stuck for rooting, they were inspected for the presence of GHWF. The cuttings were essentially free of immature stages of the GHWF and $<10$ adult GHWF were observed out of 250 cuttings. 
This demonstration study showed that EF can be used on poinsettia stock plants to control GHWF populations. Compared to chemical control, EF stabilized GHWF populations at a slightly higher level. Just before cuttings are taken, pesticide applications can be made to eliminate any remaining GHWF adults. The nymph populations were concentrated on the lower leaves, which are not included in terminal stem cuttings. Thus, the high nymph populations observed in this study did not affect cutting quality. We believe that considerably fewer EF per stock plant can be released for effective GHWF control than were released in this study. Instead of three releases, more releases using fewer EF per release should be made to control more effectively the GHWF during the early stages of infestation

\section{Literature Cited}

Albert, R. 1990. Experiences with biological control measures in glasshouses in southwest Germany. Integrated Control in Glasshouses Working Group. WPRS Bul. 5:1-5.

Renuzzi, M., G. Nicoli, and G. Manzaroli. 1990. Biological control of Bemisia tabaci (Genn.) and Trialeurodes vaporariorum (Westw.) by Encarsia formosa Gahan on poinsettia. Integrated Control in Glasshouses Working Group. WPRS Bul. 5:2731.

Cooper, R.M. and R.D. Oetting. 1987. Influence of the greenhouse environment on selected pest populations and their natural enemies. Florida Entomol. 70:5965.

Delorme, R., A. Berthier, and D. Augé. 1985. The toxicity of two pyrethroids to Encarsia formosa and its host Trialeurodes vaporariorum; prospecting for a resistant strain of the parasite. Pest. Sci. 16:332336 .

Ecke, P., Jr., O.A. Matkin, and D. E. Hartley. 1990. The poinsettia manual. 3rd ed. Paul Ecke Poinsettias, Encinitas, Calif.

Gillespie, D. and D. Quiring. 1987. Yellow sticky traps for detecting and monitoring greenhouse whitefly (Homoptera: Aleyrodidac) adults on greenhouse tomato crops. J. Econ. Entomol. 80:675-679.

Heinz, K.M. and M.P. Parrella. 1990. Losing the battle with bugs? Greenhouse Grower 8(2):36-40.

McHugh, J.B. 199la. Yes, you can win the war on whiteflies. Greenhouse Grower $9(1): 71-78$.

McHugh, J.R. 1991b. Monitoring-the first line of defense. Greenhouse Grower 9(2):61-68.

McMahon, R. W., R.K. Lindquist, A. Witt, and J. Pifher. 1989. Biological control of whiteflies on poinsettia. Ohio Florists Assn. Bul.717.

Michael, A. H. 1991. Biocontrol of whitefly. Greenhouse Grower 9(9):61-63.

Parrella, M.L. 1990. Biological control in ornamentals: Status and perspectives. Integrated Control in Glasshouses Working Group. WPRS Bul. 5:161-168.

Van Roermund, H.J.W. and J.C. Van Lenteren. 1990. Simulation of the greenhouse whitefly, Trialeurodes vaporariorum and the parasitoid Encarsia formosa. Integrated Control in Glasshouses Working Group WPRS Bul. 5:185-189.

\section{Acknowledgement}

We gratefully acknowledge Paul Ecke Poinsettias, Encinitas, Calif., for the donation of rooted poinsettia cuttings. 